\title{
The Effects of Transaction Cost and Correlation of Brownian Motions on an Insurer's Optimal Investment Strategy through Logarithmic Utility Optimization under Modified Constant Elasticity of Variance (M-CEV) Model
}

\author{
Silas A. Ihedioha ${ }^{*}$, Gbenga M. Ogungbenle², Philip T. Ajai1 \\ ${ }^{1}$ Department of Mathematics, Plateau State University, Bokkos, Nigeria \\ ${ }^{2}$ Department of Actuarial Science, University of Jos, Jos, Nigeria \\ Email: ^silasihedioha@yahoo.com, gbengarising@gmail.com, philipajai2k2@yahoo.com
}

How to cite this paper: Ihedioha, S.A., Ogungbenle, G.M. and Ajai, P.T. (2020) The Effects of Transaction Cost and Correlation of Brownian Motions on an Insurer's Optimal Investment Strategy through Logarithmic Utility Optimization under Modified Constant Elasticity of Variance (M-CEV) Model. Open Access Library Journal, 7: e6488.

https://doi.org/10.4236/oalib.1106488

Received: May 31, 2020

Accepted: July 10, 2020

Published: July 13, 2020

Copyright $\odot 2020$ by author(s) and Open Access Library Inc.

This work is licensed under the Creative Commons Attribution International License (CC BY 4.0).

http://creativecommons.org/licenses/by/4.0/

\begin{abstract}
In this work, we tackled an optimal investment strategy problem of an insurance investor, who had logarithmic utility preference and invested in two assets: 1) a riskless bond with a constant rate of return and 2) a risky asset (stock) whose price dynamics followed modified constant elasticity of variance (M-CEV) model. We focused on getting an optimal investment strategy that will maximize his returns and pays policy holders their claims whenever they occur. We derived formulae that allowed us to analyze the impact of the models parameters of the coefficient of correlation of the Brownian motions and transaction cost. It was found, among others, that if the Brownian motions increase or decrease together, the investor will need less funds to be in business than when the Brownian motions do not increase or decrease together.
\end{abstract}

\section{Subject Areas \\ Financial Mathematics}

\section{Keywords}

Effects of Transaction Cost, Insurer, Logarithmic Utility Function, Modified Constant Elasticity of Variance (M-CEV) Model, Optimal Portfolio Strategy

\section{Introduction}

Optimal portfolio problem is of practical importance in finance and insurance 
mathematics. These days, insurance companies invest both in the money market and stocks. Due to the high risks involved in the stock market, investment strategies and risk management are becoming more important.

Most of the studies and insurance mathematics have focused on finding optimal investment strategies that minimized the probability of ruin when the risk process of an insurance company follows the Cramar-Lundberg model, however, this does not come as easily as it presents difficult numerical computations of the ruin probability.

In this work therefore, we intend to study the risk process of insurance company that follows Modified constant of elasticity (MCEV) model. We take this approach, because we viewed that analytical solutions can be obtained with less difficulty. The company is expected to invest in two assets: a risk-free bond having a constant rate of return and a risky asset (stock) whose price dynamics follows Modified Constant Elasticity of variance model. The investment strategy will help in deciding how the investor should invest in both assets (the risk-free bond and the risky stock) subject to paying policy holders their claims.

\section{Review of Related Literature}

In order to achieve this objective some works done in this area were reviewed and the contributions were penciled down in the sequel.

Bayraktar (2009) [1] worked on a problem Involving individual consumers and especially beneficiaries of endowments funds who generally employ strategies such that consumption never decreases (Ratcheted) or at least they try to do this. They assumed that an agent's rate of consumption is ratcheted that is it forms a non-decreasing process. They found that the agents invests in a financial market with one risk-less asset and one risky asset with the latter's price following geometric Brownian motion as in Black schools model given the rate of consumption of the agent, they act as financial advisers and find optimal investment strategies for the agent who wishes to minimize his/her probability of running out of money either before dying or before the organization holding the endowment fails due to causes other than the ruin of the fund itself. They solved this minimization problem using stochastic optimal control techniques.

Qian and Lin (2009) [2] considered an insurance company whose surplus (reverse) is modeled by a jump diffusion risk process. The insurance company can invest part of its surplus in risky assets and purchase a proportional reinsurance for claims.

Their main goal is to find an optimal investment and proportional reinsurance policy which minimizes ruin probability. They apply stochastic control theory to solve the problem. They obtained close form expression for the minimum probability, optimal investment and proportional reinsurance policy. They found out that the minimum ruin probability satisfies the Lundberg equality. They also investigated the diffusion volatility parameter. The market price of risk and the correlation coefficient on the minimal ruin probability, optimal in- 
vestment and proportional reinsurance policy through numerical calculations.

Azcue et al. (2009) [3] considered that the reserve of an insurance company follows a Cramer-Lungberg process. They considered that the management of an insurance company had the possibility of investing part of the reserve in a risky asset. They considered that the risky asset was a stock as it is with most of the rest of the studies whose price process was a geometric Brownian motion. Their main aim was to find a dynamic choice of investment policy which would minimize the ruin probability of the insurance company. They imposed that the proportion of the reserve invested in the risky asset was to be smaller than a given positive bound for instance the case $\mathrm{a}=1$ meant that the company could not borrow money to buy stocks.

They characterized the optimal value function as the classical solution of the associated Hamilton-Jacobi-Bellman equation which was a non-linear second order integro-differential equation.

Jung (2012) [4] "Optimal investment strategies for the HARA utility under the constant elasticity of variance model", gave an explicit expression for the optimal investment strategy, under the constant elasticity of variance model, which maximizes the expected HARA utility of the final value of the surplus at the maturity time. To do this, the corresponding HJB equation was transformed into a linear partial differential equation by applying a Legendre transform and proved that the optimal investment strategy corresponding to the HARA utility function converges as to the one corresponding to the exponential utility function.

Zhao and Rong (2012) [5] "Portfolio selection problem with multiple risky assets under the constant elasticity of variance model," their work focuses on the constant elasticity of variance model for studying the utility maximization portfolio selection problem with multiple risky assets and a risk-free asset. The Hamilton-Jacobi-Bellman (HJB) equation associated with the portfolio optimization problem is established. By applying a power transform and a variable change technique, they derive the explicit solution for the constant absolute risk aversion (CARA) utility function when the elasticity coefficient is -1 or 0 . In order to obtain a general optimal strategy for all values of the elasticity coefficient, they propose a model with two risky assets and one risk-free asset and solve it under a given assumption. Furthermore, they analyzed the properties of the optimal strategies and discuss the effects of market parameters on the optimal strategies. Finally, they presented a numerical simulation to illustrate the similarities and differences between the results of the two models proposed in their work.

Ihedioha (2014) [6] worked on a problem on how to take the risk reserve of an insurance company to follow Brownian motion with drift and tackle an optimal portfolio selection problem of the company. The investment case considered was insurance company that trades two assets; the money market account (bond) growing at a rate " $r$ " and a risky stock with investment behavior in the pressure of a stochastic cash flow or a risk process continuously in the economy. His focus was on obtaining investment strategies that are optimal in the sense of opti- 
mizing the returns of the company. He established among others that the optimized investment is the assets and the optimal value functions are dependent on horizon and the wealth.

Muravey (2018) [7] work on optimal investment problem under modified constant elasticity of variance (M-CEV) model for the assets price and power utility over the final wealth for a finite horizon agent. This model was introduced in Health and Platen (2002) and is a natural extension of the famous CEV model.

From the discussions above, we note that the risk process of an insurance company in most of the papers is modeled by the Cramer-Lundberg model while investment is done with either two assets or with a single asset and reinsurance hence our choice.

We here find an optimal investment strategy for an insurance investor who trades two assets; a riskless bond with a constant rate of return and a risky asset which price dynamics follows the modified constant elasticity of variance model, and look into the effects of transaction cost and the correlation of Brownian motions on the investor's optimal strategies.

\section{Methodology}

In this section, we explain some concepts that we hope will make clear our discussion. We give brief notes on the following: Brownian motion, Geometric Brownian motion, Modified-constant elasticity of variance, Ito's lemma and formulate the insurance investor's investment problem.

\subsection{Brownian Motion}

The Brownian motion was developed to explain the random movement seen in suspended particles but is used to explain market fluctuations and stock market trading today. Brownian motion is a continuous time stochastic process (Wiener process) named in honor of Norbert Wiener and is characterized by the following four facts:

1) $Z_{0}=0$. 2) $Z_{1}$ is almost surely continuous. 3) $Z_{t}$ has independent increments. 4) $Z_{t}-Z_{s} \sim N(0, t-s)$ (for $\left.0 \leq s \leq t\right)$.

$N\left(\mu, \delta^{2}\right)$ denotes the normal distribution with the expected value $\mu$ and variance $\delta^{2}$. The condition that it has independent increment means that if $0 \leq s_{1} \leq t_{1} \leq s_{2} \leq t_{2}$ then $Z_{t_{1}}-Z_{s_{2}}$ are independent random variables.

\subsection{The Geometric Brownian Motion (GBM) (also Known as Exponential Brownian Motion)}

GBM is a continuous-time stochastic process in which the logarithm of the randomly varying quantity follows a Brownian motion with drift. It is an important example of stochastic process satisfying a stochastic differential equation (SDE). In particular, it is used in mathematical finance to model stock prices in the Black-Scholes model. 
A stochastic process $S(t)$ is said to follow GBM if it satisfies the following stochastic differential equation (SDE): $\frac{\mathrm{d} S(t)}{S(t)}=\mu \mathrm{d} t+\delta \mathrm{d} Z(t)$, where $Z(t)$ is a standard Brownian motion, $\mu$ is the appreciation rate and $\delta$ the volatility, are constants.

\subsection{Constant Elasticity of Variance Model (CEV)}

The Constant Elasticity of Variance (CEV) model is a stochastic volatility model, which generally has negative correlation between an asset return and its changes of volatility. The model was introduced by John Cox (1975) as one of the early alternatives to the geometric Brownian motion to model asset price process. The CEV model describes a process which evolves according to the following stochastic differential equation: $\frac{\mathrm{d} S(t)}{S(t)}=\mu \mathrm{d} t+\delta S^{\gamma}(t) \mathrm{d} Z(t)$, where $\mu$ and $\delta$ are constant parameters which satisfy the condition $\mu \geq 0, \delta \geq 0$. The parameter $\gamma$ controls the relationship between volatility and price of the risky asset and is a central feature of the model. If $\gamma<1$, we see the so-called leverage effect, commonly observed in equity markets where the volatility of a stock increases as its price falls.

Conversely, when $\gamma>1$, we obtain the so-called inverse leverage effect in a commodity market whereby the volatility of the price of a commodity tends to increase as its price increases.

\subsection{Modified Constant Elasticity of Variance (M-CEV)}

Consider a simple market consisting of a risk-free bond which price we shall denote by $B(t)$ and a risky asset (stock) with price $S(t)$ at time $t$. The bond and stock prices are driven by the differential equation

$$
\mathrm{d} B(t)=r(t) B(t) \mathrm{d} t ; B(t)=B>0 ;
$$

for the risk-free asset and the stochastic differential equation (SDE)

$$
\frac{\mathrm{d} S(t)}{S(t)}=[r(t)-q(t)+\lambda(S(t), t)] \mathrm{d} t+\sigma(S(t), t) \mathrm{d} Z(t) ; S(t)=S>0 ;
$$

where $Z(t)$ is a standard Wiener process, $r(t) \geq 0, q(t) \geq 0, \sigma(S, t)>0$ and $\lambda(S, t) \geq 0$ are the time-dependent risk-free interest rate, the time-dependent dividend yield, the time and state-dependent instantaneous stock volatility, and the time-and state-dependent default intensity, respectively. The M-CEV model has the following specifications:

$\sigma(S(t), t)=a S^{\gamma}(t), \quad \lambda(S(t), t)=b+c \sigma^{2}(S(t), t)=b c a^{2} S^{2 \gamma}(t), \quad q(t)=q$, $r(t)=r ; \alpha=r-q+b$; and defined by this corresponding SDE;

$\frac{\mathrm{d} S(t)}{S(t)}=\left[\alpha+c a^{2} S^{2 \gamma}\right] \mathrm{d} t+a S^{\gamma} \mathrm{d} W(t)$.

Heath and Platen (2002) considered model above with $c=1$. The case of $c \neq 1$ is not an extension of original M-CEV model because this case can be re- 
duced to the original model by a simple change of measure.

\subsection{Ito's lemma}

Recall from Taylors series;

$$
f(x)=f(0)+f^{\prime}(0) x+\frac{f^{\prime \prime}(0)}{2 !} x^{2}+\frac{f^{\prime \prime \prime}(0)}{3 !} x^{3}+\cdots
$$

This defines the case of one variable.

Now we consider the second dimension case of two variables thus:

$$
\begin{aligned}
f(a, b)= & f(0,0)+f_{a}(0,0) a+f_{b}(0,0) b+\frac{f_{a a}(0,0)}{2 !} a^{2} \\
& +\frac{f_{b b}(0,0)}{2 !} b^{2}+(0,0)+\cdots
\end{aligned}
$$

We note that

$$
f_{a b}=f_{b a} .
$$

From the above equation we get:

$$
f(a, b)=f(0,0)+f_{a} \cdot a+f_{b} \cdot b+\frac{f_{a a}}{2 !} \cdot a^{2}+\frac{f_{b b}}{2 !} \cdot b^{2}+f_{a b} \cdot a b+\cdots
$$

and

$$
\mathrm{d} f\left(t, X_{t}\right)=\frac{\partial f}{\partial t} \cdot \mathrm{d} t+\frac{\partial f}{\partial x} \cdot \mathrm{d} X(t)+\frac{1}{2} \frac{\partial^{2} v}{\partial w^{2}}(\mathrm{~d} X(t))^{2}+\frac{\partial^{2} f}{\mathrm{~d} t \mathrm{~d} x} \cdot \mathrm{d} t \cdot \mathrm{d} X(t)+\cdots
$$

Using the hint that

$$
\mathrm{d} t \mathrm{~d} t=\mathrm{d} X(t) \mathrm{d} t=\mathrm{d} t \mathrm{~d} X(t)=0, \quad \mathrm{~d} X(t) \mathrm{d} X(t)=\mathrm{d} t,
$$

then

$$
\mathrm{d} f(t, X(t))=\frac{\partial f}{\partial t} \cdot \mathrm{d} t+\frac{\partial f}{\partial x} \cdot \mathrm{d} X(t)+\frac{1}{2} \frac{\partial^{2} f}{\partial x^{2}}(\mathrm{~d} X(t))^{2}+\cdots
$$

Now comparing $\mathrm{d} f(t, X(t))$ with the function $\mathrm{d} V(t, W(t))$, we obtain the Ito's lemma,

$$
\mathrm{d} V(t, W(t))=\frac{\partial V}{\partial t} \cdot \mathrm{d} t+\frac{\partial V}{\partial W} \cdot \mathrm{d} W(t)+\frac{1}{2} \frac{\partial^{2} V}{\partial W^{2}}(\mathrm{~d} W(t))^{2} .
$$

\subsection{Model Formulation}

We adapt the formulation of Osu et al. (2014). The insurance investor trades two assets, a bond and a stock, continuously in the economy where the first asset is the money market account (bond) growing at a rate, $r$. The equation governing the dynamics of the money market account (bond) is given as

$$
\mathrm{d} B(t)=r B(t) .
$$

We assume that the risky stock available for investment has the price $S(t)$ at time $t$ is driven by Modified Constant Elasticity of Variance (M-CEV).

$$
\mathrm{d} S(t)=S(t)[r(t)-q(t)+\lambda(s, t)]+\delta(s, t) \mathrm{d} Z^{(2)}(t)
$$


where, $Z^{(2)}(t)$ is a standard Wiener process, $\delta(s, t)$ is the time-and state dependent instantaneous stock volatility, $\lambda(s, t)$ is the time - and state - dependent default intensity, $r(t)$ the time - dependent risk - free interest rate, $q(t)$ the time - dependent dividend yield.

Using the following specifications of the M-CEV model $\delta(s, t)=a S^{\gamma}, \lambda(s, t)=b+c a^{2}(s, t)=b+c a^{2}(s, t)=b+c a^{2} S^{2 \gamma}, q(t)=q$, $r(t)=r, \alpha=r-q+b$, we have the correspond stochastic differential equation (SDE)

$$
\mathrm{d} S(t)=S(t)\left[\alpha+c a^{2} S^{2 \gamma}\right] \mathrm{d} t+a S^{\gamma} \mathrm{d} Z^{2}(t)
$$

where $\alpha$ is the appreciation rate of the risky asset, $\gamma$ is the elasticity parameter of the local volatility, $c$ is the local volatility scale parameter, $\mathrm{d} Z^{(2)}(t)$ is standard Brownian motion parameter.

This work concerns an investment behavior in the presence of stochastic cash flow or a risk process which we will denote by $R(t)$ and satisfies the stochastic differential equation (SDE).

$$
\mathrm{d} R(t)=\varnothing \mathrm{d} t+\beta \mathrm{d} Z^{(1)}(t)
$$

where $\varnothing$ and $\beta$ are constants with $\beta \geq 0$. We also allow the two Brownian motions $Z^{1}$ and $Z^{2}$ to correlate with correlation coefficient $\rho$. That is $E\left(Z^{1} Z^{2}\right)=\rho$.

Let $W(t)$ be the total wealth the insurance investor has for investment and is allocated as follows: $\pi(t)$ be the total amount of the insurance investor's wealth that is invested in risky assets. The remaining balance $(W(t)-\pi(t))$ is invested in a risk - less asset (bond/market).

The wealth process of the insurance investor evolves according to the stochastic differential equations (SDE).

\subsubsection{The Case of No Transaction Cost}

When there is no transaction cost and the Brownian motions correlate, the wealth process of the insurance investor evolves according to the stochastic differential equations (SDE)

$$
\mathrm{d} W^{\pi}(t)=\pi(t) \frac{\mathrm{d} s(t)}{s(t)}+[W(t)-\pi(t)] \frac{\mathrm{d} B(t)}{B(t)}+\mathrm{d} R(t) .
$$

Applying Equation (1), (3) and (4) in (5) gives

$$
\begin{aligned}
\mathrm{d} W^{\pi}(t)= & \pi(t)\left[\left(\alpha+c a^{2} S^{2 \gamma}(t)\right) \mathrm{d} t+a S^{\gamma}(t) \mathrm{d} Z^{(2)}(t)\right] \\
& +[W(t)-\pi(t)] r \mathrm{~d} t+\varnothing \mathrm{d} t+\beta \mathrm{d} Z^{(1)}(t)
\end{aligned}
$$

Equation (6) becomes

$$
\begin{aligned}
\mathrm{d} W^{\pi}(t)= & {\left[\pi(t) \alpha+\pi(t) c a^{2} S^{2 \gamma}(t)+r(W(t)-\pi(t))+\varnothing\right] \mathrm{d} t } \\
& +\pi(t) a S^{\gamma}(t) \mathrm{d} Z^{(2)}(t)+\beta \mathrm{d} Z^{(1)}(\mathrm{d} t)
\end{aligned}
$$

Applying the assumption that $Z^{(1)}$ and $Z^{(2)}$ are correlated with correlation 
coefficient $\rho$. The quadratic variation of the wealth process is

$$
\left(\mathrm{d} W^{\pi}(t)\right)^{2}=\left[\pi^{2}(t) a^{2}\left(S^{\gamma}(t)\right)^{2}+2 \pi(t) a S^{\gamma}(t) \beta \rho+\beta^{2}\right] \mathrm{d} t .
$$

Therefore, the insurance investor's problem can be written as

$$
V(T, W)=\sup (\pi) E\left[U\left(W^{\pi}(t)\right)\right]
$$

subject to (7);

$$
\begin{aligned}
\mathrm{d} W^{\pi}(t)=[ & {\left[\pi(t) \alpha+\pi(t) c a^{2} S^{2 \gamma}(t)+r(W(t)-\pi(t))+\varnothing\right] \mathrm{d} t } \\
& +\pi(t) a S^{\gamma}(t) \mathrm{d} Z^{(2)}(t)+\beta \mathrm{d} Z^{(1)}(\mathrm{d} t)
\end{aligned}
$$

\subsubsection{The Case of Transaction Cost}

In this case, it is assumed that transaction cost is charged on the risky asset only. Therefore Equation (5) modifies to

$$
\mathrm{d} W^{\pi}(t)=\pi(t)(1-b(t)) \frac{\mathrm{d} S(t)}{S(t)}+[W(t)-\pi(t)] \frac{\mathrm{d} B(t)}{B(t)}+\mathrm{d} R(t)
$$

where $b(t)$ is the rate at which transaction cost is charged.

Applying (1), (3) and (4) to Equation (10), we get

$$
\begin{aligned}
\mathrm{d} W^{\pi}(t)= & \pi(t)[1-b t]\left[\left(\alpha+c a^{2} S^{2 \gamma}(t)\right) \mathrm{d} t+a S^{\gamma}(t) \mathrm{d} Z^{(2)}(t)\right] \\
& +[W(t)-\pi(t)] r \mathrm{~d} t+\varnothing \mathrm{d} t+\beta \mathrm{d} Z^{(1)}(t)
\end{aligned}
$$

and we further get

$$
\begin{aligned}
\mathrm{d} W^{\pi}(t) \\
=\left[(1-b(t)) \pi(t) \alpha+(1-b(t)) \pi(t) c a^{2} S^{2 \gamma}(t)+(W(t)-\pi(t)) r+\varnothing\right] \mathrm{d} t \\
+(1-b(t)) \pi(t) a S^{\gamma}(t) \mathrm{d} Z^{(2)}(t)+\beta \mathrm{d} Z^{(1)}(t)
\end{aligned}
$$

The quadratic variation of the wealth process given in Equation (12), $Z^{(1)}$ and $Z^{(2)}$ being correlated with correlation coefficient $\rho$ is

$\left(\mathrm{d} W^{\pi}(t)\right)^{2}=\left[(1-b(t))^{2} \pi^{2}(t) a^{2}\left(S^{\gamma}(t)\right)^{2}+2(1-b(t)) \pi(t) a S^{\gamma}(t) \beta \rho+\beta^{2}\right] \mathrm{d} t(13)$

Therefore, the insurance investor's problem can be written as

$$
V(t, W)=\sup (\pi) E\left[U\left(W^{\pi}(t)\right)\right]
$$

subject to (12).

\section{The Optimization Programmme}

In this section, we find the optimal investment strategies of the insurance investor's and examine the effects of transaction cost and the correlation of the Brownian motions under logarithmic utility preference. To derive the Hamilton-Jacobi Bellman (HJB) partial differential equation, we start with the Bellman equation.

The cases that will be examined are:

1) when there is no transaction cost and the Brownian motions do not correlate

2) when there is no transaction cost and the Brownian motions correlate. 
3) when there is transaction cost and the Brownian motions do not correlate

4) when there is transaction cost and the Brownian motions correlate.

The Hamilton-Jacobi-Bellman (HJB) equation is

$$
\begin{aligned}
& V_{t}+\pi(t) \alpha+\pi(t) C a^{2} s^{2 \gamma}+r(w(t)-\pi(t))+\alpha V_{w} \\
& +\left[\left(\pi(t) a s^{\gamma}(t)\right)^{2}+2 \pi(t) a s^{\gamma}(t) \beta \rho \mathrm{d} t+\beta^{2}\right] \frac{1}{2} V_{w w}=0
\end{aligned}
$$

where $V_{t}$ and $V_{w}$ are first partial derivatives of $V$ with respect to $t$ and $W$ respectively. $V_{w w}$ is second partial derivative of $V$ with respect to $W$.

We now consider the logarithm preference of the form

$$
U(W)=\ln W,
$$

and let the value function be of the form

$$
V(W, T ; t)=g(t, T) \ln W,
$$

such that at the terminal time $T$

$$
g(T ; T)=1 .
$$

We obtain from (16) that

$$
V_{t}=\ln W g_{t}, V_{w}=\frac{1}{w} g, V_{w w}=-\frac{1}{w^{2}} g
$$

The application of (16) and (18) in (14) gives

$$
\begin{aligned}
& \ln W g_{t}+\left[\pi(t) \alpha+\pi(t) C a^{2} s^{2 \gamma}(t)+r(W(t)-\pi(t))+\alpha\right] \frac{1}{w} g \\
& +\left[(\pi(t))^{2}\left(a s^{\gamma}(t)\right)^{2}+2 \pi(t) a s^{\gamma} \beta \rho+\beta^{2}\right]-\frac{1}{W^{2}} g=0
\end{aligned}
$$

To obtain the optimal value $\pi^{*}(t)$ of $\pi(t)$, we differentiate Equation (19) with respect to $\pi(t)$ and evaluate to obtain

$$
\frac{1}{W} g\left[\left(\alpha+C a^{2} s^{2 \gamma}(t)\right)-r\right]-\frac{1}{2 W^{2}} g\left[2 \pi(t) a^{2}\left(s^{\gamma}(t)\right)^{2}+2 a s^{\gamma} \beta \rho\right]=0 .
$$

From (20) we obtain the investor's optimal strategy when there is no transaction cost and the Brownian motions correlate as

$$
\pi_{n t c b}^{*}(t)=\frac{w\left(\left(\alpha+C a^{2} s^{2 \gamma}(t)\right)-r\right)}{a^{2}\left(s^{\gamma}(t)\right)^{2}}-\frac{\beta \rho}{a\left(s^{\gamma}(t)\right)} .
$$

Next, we find the general optimal investment strategy for the investor under logarithm utility preference when there is transaction cost the Brownian motions correlate

We apply Equation (12) and Equation (13) in (viii), the Ito's lemma, to obtain

$$
\begin{aligned}
\mathrm{d} V= & \frac{\partial V}{\partial t} \mathrm{~d} t+\frac{\partial V}{\partial W}\left[\left(\pi(t) \alpha+\pi(t) C a^{2} s^{2 \gamma}(t)\right)-\pi(t) b(t) \alpha-\pi(t) b(t) C a^{2} s^{2 \gamma}(t)\right. \\
& +w(t)-\pi(t) r+\theta] \mathrm{d} t+(1-b(t)) \pi(t) a s^{\gamma}(t) \mathrm{d} Z^{(2)}(t)+\beta \mathrm{d} Z^{(1)}(\mathrm{d} t) \\
& +\frac{1}{2} \frac{\partial^{2} V}{\partial W^{2}}\left[(1-b(t))^{2} \pi^{2}(t) a^{2}\left(s^{\gamma}(t)\right)^{2}+2(1-b(t)) \pi(t) a s^{\gamma}(t) \beta \rho+\beta^{2}\right] \mathrm{d} t
\end{aligned}
$$


The application of Equation (22) to Equation (14) we get the HJB equation

$$
\begin{aligned}
& V_{t}+(1-b(t)) \pi(t) \alpha+\pi(t) C a^{2} s^{2 \gamma}(t) \\
& -\pi(t) b(t) C a^{2} s^{2 \gamma}(t)+(w(t)-\pi(t)) r+\theta V_{w} \\
& +\left[(1-b(t))^{2}(\pi(t))^{2}\left(a s^{\gamma}(t)\right)^{2}+2(1-b(t)) \pi(t) a s^{\gamma}(t) \beta \rho+\beta^{2}\right] \frac{1}{2} V_{w w}=0
\end{aligned}
$$

Using (15) to (17) in Equation (23) we obtain.

$$
\begin{aligned}
& \ln w g_{t}+\left[(1-b(t)) \pi(t) \alpha+\pi(t) C a^{2} s^{2 \gamma}(t)\right. \\
& \left.-\pi(t) b(t) C a^{2} s^{2 \gamma}(t)+r(w(t)-\pi(t))+\theta\right] \frac{1}{w} g \\
& -\frac{1}{w^{2}} g\left[(1-b(t))(\pi(t))^{2}\left(a s^{\gamma}(t)\right)^{2}+2(1-b(t)) \pi(t) a s^{\gamma}(t) \beta \rho+\beta^{2}\right]=0
\end{aligned}
$$

Differentiating Equation (24) with respect to $\pi(t)$ we get

$$
\begin{aligned}
& \frac{1}{w} g\left[\left((1-b(t)) \alpha+C a^{2} s^{2 \gamma}(t)\right)-b(t) C a^{2} s^{2 \gamma}(t)-r\right] \\
& -\frac{1}{2 w^{2}} g\left[2 \pi(t)(\alpha-b(t))^{2} a^{2}\left(s^{\gamma}(t)\right)^{2}+2(1-b(t)) a s^{\gamma}(t) \beta \rho\right]=0
\end{aligned}
$$

from which on making $\pi(t)$ the subject we obtain the investor's optimal strategy when there is transaction cost and correlated Brownian motions as

$$
\begin{aligned}
\pi_{t c b}^{*}(t)= & \frac{w\left(\alpha+C a^{2} s^{2 \gamma}(t)-b(t) \alpha-b(t) C a^{2} s^{2 \gamma}(t)-r\right)}{a^{2}(\alpha-b(t))^{2}\left(s^{\gamma}(t)\right)^{2}} \\
& -\frac{(1-b(t)) \beta \rho}{a(\alpha-b(t))^{2}\left(s^{\gamma}(t)\right)}
\end{aligned}
$$

Now we look at the listed cases thus:

Case 1: when there is no transaction cost and the Brownian motions do not correlate

In (26)

$$
\pi_{n t c b}^{*}(t)=\frac{w\left(\left(\alpha+C a^{2} s^{2 \gamma}(t)\right)-r\right)}{a^{2}\left(s^{\gamma}(t)\right)^{2}}-\frac{\beta \rho}{a\left(s^{\gamma}(t)\right)},
$$

if $\rho=0$ and there is no transaction cost, the optimal strategy is

$$
\pi_{n t}^{*}(t)=\frac{w\left(\left(\alpha+C a^{2} s^{2 \gamma}(t)\right)-r\right)}{a^{2}\left(s^{\gamma}(t)\right)^{2}} .
$$

The optimal investment strategy is a fraction the wealth at hand and depends on horizon. The investor remains in business as long as $\alpha+\mathrm{Ca}^{2} s^{2 \gamma}(t)>r$.

Case 2: when there is no transaction cost and the Brownian motions correlate.

In this case the insurance investor's optimal strategy is as given by equation (21) 


$$
\pi_{n t c c b}^{*}(t)=\frac{w\left(\left(\alpha+C a^{2} s^{2 \gamma}(t)\right)-r\right)}{a^{2}\left(s^{\gamma}(t)\right)^{2}}-\frac{\beta \rho}{a\left(s^{\gamma}(t)\right)} .
$$

Clearly

$$
\pi_{n t c c b}^{*}(t)=\pi_{n c t}^{*}(t)-\frac{\beta \rho}{a\left(s^{\gamma}(t)\right)} .
$$

This implies that the investor requires fewer funds to invest in the risky asset when the Brownian motions have positive correlation. That is, if the Brownian motions increase or decrease together the investor will need less funds to be in business than when the Brownian motions do not correlate.

If the Brownian motions do not either increase or decrease together the reverse becomes the case as shown by: when the correlation Brownian motion is negative, say $\rho=-\theta$, then Equation (28) becomes

$$
\pi_{n t c b}^{*}(t)=\pi_{n c t}^{*}(t)+\frac{\beta \theta}{a s^{\gamma}(t)} .
$$

Case 3: when there is transaction cost and the Brownian motions do not correlate

Here we have from Equation (26)

$$
\begin{aligned}
\pi_{\text {tccb }}^{*}(t)= & \frac{w\left(\alpha+C a^{2} s^{2 \gamma}(t)-b(t) \alpha-b(t) C a^{2} s^{2 \gamma}(t)-r\right)}{a^{2}(\alpha-b(t))^{2}\left(s^{\gamma}(t)\right)^{2}} \\
& -\frac{(1-b(t)) \beta \rho}{a(\alpha-b(t))^{2}\left(s^{\gamma}(t)\right)}
\end{aligned}
$$

that if $\rho=0$ there is no correlation of Brownian motions and there is transaction cost, then the optimal strategy becomes

$$
\pi_{t c}^{*}(t)=\frac{w\left(\alpha+C a^{2} s^{2 \gamma}(t)-b(t) \alpha-b(t) C a^{2} s^{2 \gamma}(t)-r\right)}{a^{2}(\alpha-b(t))^{2}\left(s^{\gamma}(t)\right)^{2}} .
$$

It can be seen that

$$
\pi_{t c c b}^{*}(t)=\pi_{t c}^{*}(t)-\frac{(1-b(t)) \beta \rho}{a(\alpha-b(t))^{2}\left(s^{\gamma}(t)\right)} .
$$

Equation (29) shows that the investor requires extra $\frac{(1-b(t)) \beta \rho}{a(\alpha-b(t))^{2}\left(s^{\gamma}(t)\right)}$ amount money to invest in the risky asset when there is transaction cost and the Brownian motions correlate with positive correlation coefficient. That is, if the Brownian motions increase or decrease together the investor will need less funds to be in business than when the Brownian motions do not correlate.

The reverse is the case as shown by: when the correlation Brownian motion is negative (if the Brownian motions do not either increase or decrease together), say $\rho=-\theta$, then Equation (31) becomes 


$$
\pi_{t c c b}^{*}(t)=\pi_{t c}^{*}(t)+\frac{(1-b(t)) \beta \theta}{a(\alpha-b(t))^{2}\left(s^{\gamma}(t)\right)} .
$$

Case 4: when there is transaction cost and the Brownian motions correlate

In this case, the general rule holds as in Equation (26)

$$
\begin{aligned}
\pi_{t c b}^{*}(t)= & \frac{w\left(\alpha+C a^{2} s^{2 \gamma}(t)-b(t) \alpha-b(t) C a^{2} s^{2 \gamma}(t)-r\right)}{a^{2}(\alpha-b(t))^{2}\left(s^{\gamma}(t)\right)^{2}} \\
& -\frac{(1-b(t)) \beta \rho}{a(\alpha-b(t))^{2}\left(s^{\gamma}(t)\right)}
\end{aligned}
$$

This is the situation when $\rho>0$, but when $\rho<0$, say $\rho=-\theta$, we have

$$
\begin{aligned}
\pi_{t c b}^{*}(t)= & \frac{w\left(\alpha+C a^{2} s^{2 \gamma}(t)-b(t) \alpha-b(t) C a^{2} s^{2 \gamma}(t)-r\right)}{a^{2}(\alpha-b(t))^{2}\left(s^{\gamma}(t)\right)^{2}} \\
& +\frac{(1-b(t)) \beta \theta}{a(\alpha-b(t))^{2}\left(s^{\gamma}(t)\right)}
\end{aligned}
$$

the insurance investor will require more amount of money to invest in the risky asset.

\section{Conclusions}

In this research, we take the risk reserve of an insurance company to follow modified constant elasticity of variance model to tackle an optimal problem of the company. The investment case considered was insurance company that trades two assets: the money market account (bond) growing at a rate $\mathrm{r}$ and a risky stock with investment behavior in the presence of stochastic cash flow or a risk process, continuously in the economy. Our focus was on obtaining investment strategies that are optimal in the sense of optimizing the returns of the company; we establish among others that the optimized investment in the assets and the optimal value function are dependent on horizon and wealth. It is recommended that the managers of the assets of the insurance company should take into consideration this horizon dependency when making policy decision.

In this study, we optimize the insurance company's returns under the logarithm utility function. The Hamilton-Jacobi-Bellman (HJB) partial differential equation is used to solve the dynamic optimization problem.

Recent studies have uncovered several systematic patterns that increase the probability that individual investors can select stock portfolio with excess returns. In this study, the problem of investors' portfolio selection through logarithmic utility optimization under modified constant elasticity of variance model was dealt with.

The characterization of investors' behavior is central in the optimal portfolio selection decision making. The main emphasis here is on how the choice of lo- 
garithmic utility preference affects the insurance investor's investment choices. The proportion for optimizing the company's expected return was observed to be a proportion of the investor's total wealth.

\section{Acknowledgements}

We thank God for empowering us to do this work. We wish to thank the authorities of Plateau State University and the University of Jos where we serve. Our gratitude goes to all colleagues whom we have worked and shall work with.

\section{Conflicts of Interest}

The authors declare no conflicts of interest regarding the publication of this paper.

\section{References}

[1] Bayraktar, E. and Young, R.V. (2008) Minimizing the Probability of Ruin When the Consumption Is Ratcheted. North American Actuarial Journal, 12, 428-442. https://doi.org/10.1080/10920277.2008.10597535

[2] Qian, Y. and Lin, X. (2009) Ruin of Probabilities under an Optimal Investment and Proportional Reinsurance Policy in Jump Diffusion Risk Process. ANZIAMI, 24, 34-48. https://doi.org/10.1017/S144618110900042X

[3] Azcue, P. and Muler, M. (2009) Optimal Investment Strategy to Minimize the Ruin Probability of an Insurance Company under Borrowing Constraints. Insurance Mathematics and Economics, 44, 26-34. https://doi.org/10.1016/j.insmatheco.2008.09.006

[4] Jung, K.J.H. (2012) Optimal Investment Strategies for the HARA Utility under the Constant Elasticity of Variance Model. Insurance: Mathematics and Economics, 51, 674-684. https://doi.org/10.1016/j.insmatheco.2012.09.009

[5] Zhao, H. and Rong, X. (2012) Portfolio Selection Problem with Multiple Risky Assets under the Constant Elasticity of Variance Model. Insurance: Mathematics and Economics, 50, 179-190. https://doi.org/10.1016/j.insmatheco.2011.10.013

[6] Ihedioha, S.A. (2014) Optimization of Investment Returns of Insurance Company under Power Utility Function. Inventi Impact. Business Research and Reviews, No. $1,1-7$.

[7] Muravey, D. (2018) Optimal Portfolio Management in a Modified Constant Elasticity of Variance Model. Computational Mathematics and Modeling, 29, 110-119. https://doi.org/10.1007/s10598-018-9393-6 